Bull. Korean Math. Soc. 49 (2012), No. 4, pp. 705-714

http://dx.doi.org/10.4134/BKMS.2012.49.4.705

\title{
MATHEMATICAL ANALYSIS OF NONLINEAR DIFFERENTIAL EQUATION ARISING IN MEMS
}

\author{
RUIFENG ZHANG AND NA Li
}

\begin{abstract}
In this paper, we study nonlinear equation arising in MEMS modeling electrostatic actuation. We will prove the local and global existence of solutions of the generalized parabolic MEMS equation. We present that there exists a constant $\lambda^{*}$ such that the associated stationary problem has a solution for any $\lambda<\lambda^{*}$ and no solution for any $\lambda>\lambda^{*}$. We show that when $\lambda<\lambda^{*}$ the global solution converges to its unique maximal steady-state as $t \rightarrow \infty$. We also obtain the condition for the existence of a touchdown time $T \leq \infty$ for the dynamical solution. Furthermore, there exists $p_{0}>1$, as a function of $p$, the pull-in voltage $\lambda^{*}(p)$ is strictly decreasing with respect to $1<p<p_{0}$, and increasing with respect to $p>p_{0}$.
\end{abstract}

\section{Introduction}

Electrostatic actuation is crucial for micro-electromechanical systems (MEMS), which are based on an electrostatic-controlled tunable capacitor, and widely used in accelerometers for airbag deployment in automobiles, microresonators, switches, micro-mirrors, accelerometers, and so on. The simplicity and importance of this technique have led many applied mathematicians and engineers to study mathematical models of electrostatic-elastic interactions. A mathematical model of the physical phenomena, leading to a partial differential equation for the dimensionless dynamic deflection of the membrane, was derived and analyzed in [2] and [6]. In the damping-dominated limit, and using a narrow-gap asymptotic analysis, the dimensionless dynamic deflection $v=v(x, t)$ of the membrane on a bounded domain $\Omega$ in $\mathbb{R}^{N}(N \geq 2)$, is found to satisfy the following parabolic problem $([1,5,6,13])$

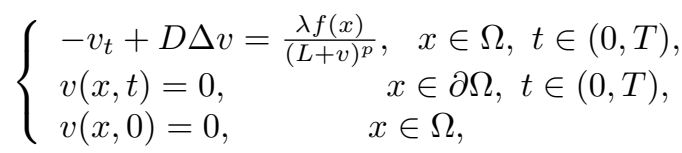

Received March 23, 2011; Revised December 27, 2011.

2010 Mathematics Subject Classification. 35K55, 35K65, 35B40.

Key words and phrases. MEMS equation, upper-and-lower solution method, global convergence, touchdown time. 
where $\lambda>0$ is a parameter, $D>0, p>1$ are fixed constants. In particular the problem with $p=2$ has been intensively studied $([5,6])$, which models a simple electrostatic MEMS device consisting of a thin dielectric elastic membrane with boundary supported at 0 above a rigid ground plate located at $-L$. Here $u$ is modeled to describe dynamic deflection of the elastic membrane. The voltage $V$ must be limited, or the elastic plate will be pulled-in.

The parameter $D>0$ is the tension constant. The parameter $\lambda$ in (1.1) characterizes the relative strength of the electro-static and mechanical forces in the system, and is proportional to the supply voltage squared $V^{2}$. We shall use here the parameter $\lambda$ (resp., $\lambda^{*}$ ) to represent the applied voltage $V$ (resp., pull-in voltage $\left.V^{*}\right)$. Referred to as the permittivity profile, $f(x)$ satisfy

$$
\left\{\begin{array}{l}
f \in C^{\alpha}(\bar{\Omega}) \text { for some } \alpha \in(0,1], 0 \leq f \leq 1 \\
f>0 \text { on a subset of } \Omega \text { of positive measure. }
\end{array}\right.
$$

Recently, Pelesko, Ghoussoub and Guo, Wang and Ruan studied in $[4,11,12]$ the steady-states of (1.1) with $p=2, p>0$, respectively, when $f(x)$ is assumed to be bounded away from zero. They established in above cases an upper bound $\bar{\lambda}_{1}$ for $\lambda^{*}$, respectively, and derived numerical results for the powerlaw permittivity profile, from which the larger pull-in voltage and thereby the larger pull-in distance, the existence and multiplicity of the steady-states were observed.

In $[5,6]$, authors studied the dynamic behavior of (1.1) with $p=2$. They considered a more general class of profiles $f(x)$, i.e., $0 \leq f(x) \leq 1$ on $\Omega$ with $f(x)>0$ on a subset of positive measure. They obtained larger pull-in voltage $\lambda^{*}$ and larger pull-in distance for different classes of varying permittivity profiles. They established upper and lower bounds on first touchdown times, and analyzed their dependence on $f, \lambda$ and $\Omega$ by applying various analytical and numerical techniques.

In [8], Hui studied the following nonlocal parabolic equation

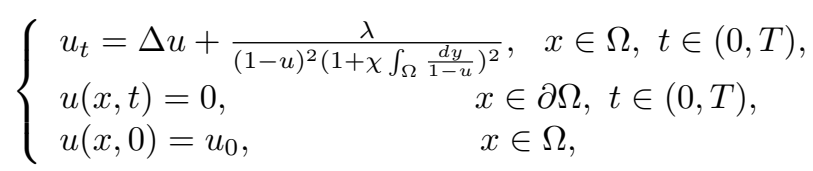

where $\chi>0$ is a constant. He obtained upper bounds for the quenching time of the solution of the nonlocal parabolic MEMS equation in $\Omega \times(0, \infty)$, when $\lambda$ is large. Furthermore, he studied the generalized parabolic MEMS equation in [7]. He obtained a series of sharp existence theorems for solution of the generalized MEMS equation.

It is often convenient to take the change of variable $u=-v$ in considering problem (1.1). Then we have

$$
\begin{cases}u_{t}-D \Delta u=\frac{\lambda f(x)}{(L-u)^{p}}, & x \in \Omega, t \in(0, T), \\ u(x, t)=0, & x \in \partial \Omega, t \in(0, T), \\ u(x, 0)=0, & x \in \Omega,\end{cases}
$$


and the associated stationary problem

$$
\begin{cases}-D \Delta u=\frac{\lambda f(x)}{(L-u)^{p}}, & x \in \Omega \\ u(x, t)=0, & x \in \partial \Omega .\end{cases}
$$

Let $Q=\{\lambda>0 \mid(1.5)$ possesses at least one solution $\}$ and the number

$$
\lambda^{*}=\lambda^{*}(\Omega, f, p)=\sup _{\lambda \in Q} \lambda .
$$

The constant $\lambda^{*}$ is called the pull-in voltage of the equation (1.5) in the literature of MEMS.

In this paper, we deal with issues of global convergence in the dynamic problem (1.4). For $p>1$, and $f(x)$ satisfies (1.2), we will prove the local and global existence and comparison theorems of solutions of the dynamic problem (1.4). We present that there exists a constant $\lambda^{*}$ such that the associated stationary problem (1.5) has a solution for any $\lambda<\lambda^{*}$ and no solution for any $\lambda>\lambda^{*}$. We show that when $\lambda<\lambda^{*}$ the global solution converges to its unique minimal steady-state as $t \rightarrow \infty$. We also obtain the condition for the existence of a touchdown time $T \leq \infty$ for the dynamical solution. Furthermore, there exists $p_{0}>1$, as a function of $p$, the pull-in voltage $\lambda^{*}(p)$ is strictly decreasing with respect to $1<p<p_{0}$, and increasing with respect to $p>p_{0}$. It is interesting that, we extend our method to study a more general model which is widely useful in applications $([1,5,6])$.

\section{Global regular solution for problem (1.4)}

In this section, we will use upper-and-lower solution method to study the existence of solutions for the parabolic equation according to the problem (1.4).

We define upper and lower solutions, $u^{+}$and $u^{-}$, if they satisfy, respectively,

$$
\begin{cases}u_{t}^{+}-D \Delta u^{+} \geq \frac{\lambda f(x)}{\left(L-u^{+}\right)^{p}}, & x \in \Omega, t \in(0, T), \\ u^{+}(x, t) \geq 0, & x \in \partial \Omega, t \in(0, T), \\ u^{+}(x, 0) \geq 0, & x \in \Omega, t=0\end{cases}
$$

and

$$
\begin{cases}u_{t}^{-}-D \Delta u^{-} \leq \frac{\lambda f(x)}{\left(L-u^{-}\right)^{p}}, & x \in \Omega, t \in(0, T) \\ u^{-}(x, t) \leq 0, & x \in \partial \Omega, t \in(0, T) \\ u^{-}(x, 0) \leq 0, & x \in \Omega, t=0\end{cases}
$$

We now invoke an iterative scheme

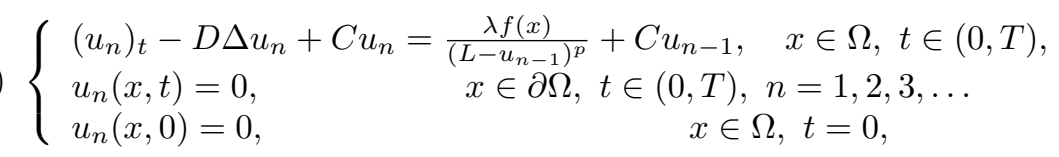

where $C>0$ is a suitable constant and $u_{0}=0$. Because $0 \leq u<L$, we can confirm that $u=0$ is the lower solution $u^{-}$. To obtain a solution of the problem 
(1.4), we assume that it has a upper solution $u^{+}$, then $u_{1}$ and $u^{+}$satisfy:

$$
\begin{aligned}
& \left(u^{+}-u_{1}\right)_{t}-D \Delta\left(u^{+}-u_{1}\right)+C\left(u^{+}-u_{1}\right) \\
\geq & \frac{\lambda f(x)}{\left(L-u^{+}\right)^{p}}-\frac{\lambda f(x)}{L^{p}}+C\left(u^{+}-u_{0}\right) \\
= & \lambda f(x) \frac{L^{p}-\left(L-u^{+}\right)^{p}}{L^{p}\left(L-u^{+}\right)^{p}}+C u^{+} \\
> & 0, \quad x \in \Omega, t \in(0, T) .
\end{aligned}
$$

Because of $u^{+}-u_{1} \geq 0, x \in \partial \Omega, t \in[0, T)$, we can use the maximum principle to obtain $u_{1} \leq u^{+}, x \in \Omega, t \in(0, T)$.

Let $u_{1}$ and $u^{-}$satisfy:

$$
\left(u_{1}-u^{-}\right)_{t}-D \Delta\left(u_{1}-u^{-}\right)+C\left(u_{1}-u^{-}\right) \geq 0, \quad x \in \Omega, t \in(0, T) .
$$

By $u_{1}-u^{-} \geq 0, x \in \partial \Omega, t \in[0, T)$, we can also use the maximum principle to obtain $u^{-} \leq u_{1}, x \in \Omega, t \in(0, T)$.

We can obtain a sequence as the same method:

$$
0=u^{-} \leq u_{1} \leq \cdots \leq u_{n-1} \leq u_{n} \leq \cdots \leq u^{+}<L .
$$

We now show that when $\lambda$ is small enough, the problem (1.4) has a upper solution. To see this, we use $k_{1}$ and $U_{1}$ to denote the first eigenvalue and the associated eigenfunction of the operate $-\Delta$ over a large bounded domain $\Omega_{1}$ containing $\bar{\Omega}$, so that $U_{1}=0$ on $\partial \Omega_{1}$ and $U_{1}>0$ in $\Omega_{1}$. Let $\varepsilon>0$ be a small number so that $0<\varepsilon U_{1}<\frac{L}{2}$. Then $-D \Delta\left(\varepsilon U_{1}\right)=\varepsilon k_{1} D U_{1}$. We need that

$$
\varepsilon k_{1} D U_{1} \geq \frac{\lambda f(x)}{\left(L-\varepsilon U_{1}\right)^{p}}, \quad x \in \Omega, t \in(0, T),
$$

thus

$$
\lambda f(x) \leq \varepsilon k_{1} D U_{1}\left(L-\varepsilon U_{1}\right)^{p},
$$

i.e.,

$$
0<\lambda^{*} \leq k_{1} D \frac{p^{p}}{(p+1)^{p+1}} \frac{L^{p+1}}{\inf _{x \in \Omega} f(x)}, \quad p>1 .
$$

If $0 \leq \lambda<\lambda^{*}$, then $\varepsilon U_{1}$ is the upper solution, i.e., $0 \leq u^{-}<\varepsilon U_{1}=u^{+}<L$.

So, we can obtain the existence of the solution of the problem (1.4) with the upper and lower solution.

Lemma 2.1. Assume $f$ is a function satisfying (1.2) on a bounded domain $\Omega$ in $\mathbb{R}^{N}$, and $p>1$. If $0 \leq \lambda<\lambda^{*}$, then there exists a global regular solution $u(x, t)$ for (1.4), where the finite voltage $\lambda^{*}=\lambda^{*}(\Omega, f, p)>0$ is as in (2.7).

Corollary 2.2. Let $0 \leq \lambda<\lambda^{*}$ and $0 \leq f \in C(\bar{\Omega})$. Suppose $u_{1}, u_{2}$ are nonnegative subsolution and supersolution of $(1.4)$ in $\Omega \times(0, T)$, respectively. Then $u_{1} \leq u_{2}$ in $\bar{\Omega} \times(0, T)$. 
Lemma 2.3. Under the condition of the Lemma 2.1, then problem (1.4) has a unique global solution.

Proof. Suppose $u_{1}$ and $u_{2}$ are solutions of (1.4) on the domain $\Omega \times[0, T]$ such that $\left\|u_{i}\right\|_{L^{\infty}(\Omega \times[0, T])}<L$ for $i=1,2$. Let $u=u_{1}-u_{2}$, then we have

$$
u_{t}-D \triangle u=g u,
$$

with initial data $u(x, 0)=0$ and zero boundary condition. Here

(2.9) $g(x, t)=\lambda f(x) \frac{\left(L-u_{2}\right)^{p-1}+\left(L-u_{2}\right)^{p-2}\left(L-u_{1}\right)+\cdots+\left(L-u_{1}\right)^{p-1}}{\left(L-u_{1}\right)^{p}\left(L-u_{2}\right)^{p}}$.

The assumption on $u_{1}, u_{2}$ implies that $g(x, t) \in L^{\infty}(\Omega \times[0, T])$. We now fix $T_{1} \in[0, T]$ and consider the solution $v$ of the problem

$$
\left\{\begin{array}{l}
v_{t}+D \Delta v+g v=0, \quad x \in \Omega, t \in\left(0, T_{1}\right) \\
v\left(x, T_{1}\right)=\xi(x) \in C_{0}(\Omega), \\
v(x, t)=0, \quad x \in \partial \Omega
\end{array}\right.
$$

The standard linear theory $[5,9]$ gives that the solution of $(2.10)$ is a unique and bounded. Now multiplying $(2.8)$ by $v$, and integrating it on $\Omega \times\left[0, T_{1}\right]$, together with (2.10), yield that

$$
\int_{\Omega} u\left(x, T_{1}\right) \xi(x) d x=0,
$$

for arbitrary $T_{1}$ and $\xi(x)$, which implies that $u=0, u_{1}=u_{2}$.

Lemma 2.4. Let $\lambda>0, p>1$, and $\Omega \subset \mathbb{R}^{N}$ be a bounded domain such that ball $B_{R}=B_{R}(0) \subset \Omega$ for some constant $R>0$. Let $0<f \in C(\bar{\Omega}), 0 \leq u<L$ be a solution of (1.4) in $\Omega \times(0, T)$ and $0<\delta \leq \inf _{x \in \Omega} f(x)$. Suppose $\lambda>\frac{2 n D}{\delta R^{2}}$. Then $T$ satisfies

$$
T \leq \frac{1}{\lambda \delta}\left(1-\frac{2 n D}{\lambda \delta R^{2}}\right)^{-1}
$$

Proof. Let $w=L-u$ and $w_{0}=L$. Then $w$ satisfies

$$
\begin{cases}w_{t}-D \Delta w+\lambda f(x) w^{-p}=0, & x \in \Omega, t \in(0, T), \\ w(x, t)=L, & x \in \partial \Omega, t \in(0, T) \\ w(x, 0)=L, & x \in \Omega .\end{cases}
$$

Let

$$
\eta(x, t)=1-\lambda \delta \gamma t\left(1-\frac{|x|^{2}}{R^{2}}\right), \quad x \in B_{R}, t \in(0, T),
$$

where $\gamma=1-\frac{2 n D}{\lambda \delta R^{2}}>0$. Then

$$
\frac{2 n D}{R^{2}} \cdot \frac{1}{\lambda \delta \gamma}=\gamma^{-1}-1 .
$$

By (2.14), we get

$$
\eta_{t}-D \Delta \eta+\lambda \delta \eta^{-p} \geq \lambda \delta \gamma\left(\gamma^{-1}-1-\frac{2 n D}{R^{2}} t\right) \geq 0, \quad x \in B_{R}, t \in\left(0, T_{1}\right)
$$


where $T_{1}=\min \left\{T, \frac{1}{\lambda \delta \gamma}\right\}$. Hence $\eta$ is a supersolution of

$$
\begin{cases}\eta_{t}-D \Delta \eta+\lambda \delta \eta^{-p}=0, & (x, t) \in B_{R} \times\left(0, T_{1}\right), \\ \eta(x, t)=L, & (x, t) \in \partial B_{R} \times\left(0, T_{1}\right), \\ \eta(x, 0)=L, & x \in \bar{B}_{R} .\end{cases}
$$
have

Suppose $T>\frac{1}{\lambda \delta \gamma}$. Since $w$ is a subsolution of (2.15), by Corollary 2.2, we

$$
0<w \leq \eta, \quad(x, t) \in \bar{B}_{R} \times\left(0, T_{1}\right) .
$$

So $0<w\left(0, \frac{1}{\lambda \delta \gamma}\right) \leq \eta\left(0, \frac{1}{\lambda \delta \gamma}\right)=0$. Contradiction arise and (2.12) follows.

Theorem 2.5. Assume $f$ is a function satisfying (1.2) on a bounded domain $\Omega$ in $\mathbb{R}^{N}$, and $p>1$. Then for any $\lambda>0$ there exists $T>0$ such that (1.4) has a unique solution $0 \leq u<L$ in $\Omega \times(0, T)$ which satisfies

(2.16) $u(x, t)=\lambda \int_{0}^{t} \int_{\Omega} G(x, y, t-s) \frac{f(y)}{(L-u(y, s))^{p}} d y d s, \quad(x, t) \in \bar{\Omega} \times(0, T)$.

Where $G(x, y, t)$ is the Dirichlet Green function for the heat equation in $\Omega \times$ $(0, T)$.

Proof. Let

$$
T=\frac{L+a}{2 \lambda\|f\|_{L^{\infty}}}\left(\frac{L-a}{2}\right)^{p}, \quad p>1,0<a<1,
$$

and

$$
u_{1}(x, t)=\lambda \int_{0}^{t} \int_{\Omega} G(x, y, t-s) \frac{f(y)}{L^{p}} d y d s, \quad(x, t) \in \bar{\Omega} \times(0, T) .
$$

Let $T_{1}=\sup \left\{0<t_{1}<T: u_{1}<\frac{L+a}{2},(x, t) \in \bar{\Omega} \times\left(0, t_{1}\right)\right\}$. Suppose $T_{1}<T$. $\forall(x, t) \in \bar{\Omega} \times\left(0, T_{1}\right]$, then $u_{1} \leq \lambda\|f\|_{L^{\infty}} \cdot \frac{t}{L^{p}}<\frac{L+a}{2}$. By continuity of $u_{1}$ there exists $0<\delta<\frac{T-T_{1}}{2}$ such that $u_{1}<\frac{L+a}{2}$ holds for all $(x, t) \in \bar{\Omega} \times\left(0, T_{1}+\delta\right]$. This contradicts the maximality of $T_{1}$. Hence $T_{1}=T$ and (2.18) holds for all $(x, t) \in \bar{\Omega} \times(0, T]$.

We define

(2.19) $u_{n+1}(x, t)=\lambda \int_{0}^{t} \int_{\Omega} G(x, y, t-s) \frac{f(y)}{\left(L-u_{n}\right)^{p}} d y d s, \quad(x, t) \in \bar{\Omega} \times(0, T)$.

Let $T_{n}=\sup \left\{0<t_{1}<T: u_{n}<\frac{L+a}{2},(x, t) \in \bar{\Omega} \times\left(0, t_{1}\right)\right\}$. By induction, we obtain $T_{n}=T$ for all $n \in \mathbb{Z}^{+}$. Then

$$
u_{n}<\frac{L+a}{2}, \quad \forall(x, t) \in \bar{\Omega} \times(0, T) .
$$

By (2.18), (2.19), (2.20) and standard parabolic theory [3], we have $u_{n} \in$ $C^{2,1}(\bar{\Omega} \times(0, T])$ for all $n \geq 2$. Then $u_{n}$ satisfies

$$
\left\{\begin{array}{l}
\frac{\partial u_{n}}{\partial t}-D \Delta u_{n}=\frac{\lambda f(x)}{\left(L-u_{n}\right)^{p}}, \quad x \in \Omega, t \in(0, T), \\
u_{n}(x, t)=0, \quad x \in \partial \Omega, t \in(0, T), \\
u_{n}(x, 0)=0, \quad x \in \Omega
\end{array}\right.
$$


By $(2.20),(2.21)$ and the Schauder estimates for the heat equation $([3,7])$ $\left\{u_{n}\right\}_{n=2}^{\infty}$ are uniformly bounded in $C^{2+\beta, 1+\frac{\beta}{2}}\left(\Omega^{\prime}\right)$ ([7]) for any compact subset $\Omega^{\prime} \subset \bar{\Omega} \times(0, T]$, where $0<\beta<1$ is some constant. By the Ascoli theorem and a diagonalization argument $\left\{u_{n}\right\}_{n=2}^{\infty}$ has a subsequence which converges uniformly in $C^{2+\beta, 1+\frac{\beta}{2}}\left(\Omega^{\prime}\right)$ to some function $u$ for any compact subset $\Omega^{\prime} \subset$ $\bar{\Omega} \times(0, T]$ as $n \rightarrow \infty$. Then $u$ satisfies $(2.16)$,

$$
u(x, t) \leq \frac{L+a}{2}<L, \quad \forall(x, t) \in \bar{\Omega} \times(0, T),
$$

and (1.4). Hence $u$ is a solution of (1.4) in $\Omega \times(0, T)$.

By an argument similar to the proof of Theorem 2.5, we have the following theorem.

Theorem 2.6. Let $p>1$ and

$$
0<f \in C^{\alpha}\left(\mathbb{R}^{N}\right) \text { for some constant } 0<\alpha<1 \text {. }
$$

Then for any $\lambda>0$ there exists a constant $T>0$ such that Cauchy problem

$$
\left\{\begin{array}{l}
u_{t}-D \Delta u=\frac{\lambda f(x)}{(L-u)^{p}}, \quad x \in \mathbb{R}^{N}, t \in(0, T), \\
u(x, 0)=0, \quad x \in \mathbb{R}^{N},
\end{array}\right.
$$

has a solution $0 \leq u<L$ in $\mathbb{R}^{N} \times(0, T)$ which satisfies

$$
u(x, t)=\lambda \int_{0}^{t} \int_{\mathbb{R}^{N}} Z(x, y, t-s) \frac{f(y)}{(L-u(y, s))^{p}} d y d s,
$$

where $Z(x, y, t)=(4 D \pi t)^{-\frac{N}{2}} e^{-\frac{|x-y|^{2}}{4 D t}}$.

\section{Global convergence at infinite time}

In this section we will prove the convergence of solution of (1.4) for any $0<\lambda<\lambda^{*}$ as $t \rightarrow \infty$. We also obtain the conditions for the solution of (1.4) to have finite touchdown time or infinite time.

Definition 3.1. If $u_{*}$ is a solution of (1.5) such that for any other solution $v$ of (1.5), one has

$$
u_{*} \leq v, \text { a.e. } \quad x \in \Omega,
$$

we say that $u_{*}$ is a unique minimal solution of (1.5).

In [10, 12], Lin and Yang, Wang and Ruan derived the equation (1.5) with $p=2, p>0$ in the study of charged plates in electrostatic actuators, respectively. Here $\lambda=k V^{2}$ where $V$ is the electric voltage and $k$ is a positive constant. For the boundary condition $\left.u\right|_{\partial \Omega}=0$, they found that there exists $0<\lambda^{*}<\infty$ such that for $\lambda \in\left(0, \lambda^{*}\right),(1.5)$ has a minimal and regular solution $u_{*}$. For $\lambda>\lambda^{*},(1.5)$ does not have any regular solution.

Similarly to the method in $[4,6,7,10,11,12]$, by the definition $\lambda^{*}(1.6)$ and the proof of Lemma 2.1 we can get: 
Lemma 3.2. Suppose $\lambda^{*}=\lambda^{*}(\Omega, f, p)>0$ is as in Lemma 2.1. Then the following hold:

1. If $0 \leq \lambda<\lambda^{*}$, there exists the regular solution of (1.5).

2. If $\lambda>\lambda^{*}$, there exists no solution of (1.5).

Proposition 3.3. Let $\Omega_{1} \subset \Omega_{2}$ and let $f_{1}, f_{2}$ satisfy (1.2) in $\Omega_{1}, \Omega_{2}$, respectively for some constant $0<\alpha<1$ such that $f_{1} \leq f_{2}$ in $\Omega_{1}$. Then $\lambda^{*}\left(\Omega_{1}, f_{1}, p\right) \geq \lambda^{*}\left(\Omega_{2}, f_{2}, p\right)$.

If $0<\lambda<\lambda^{*}\left(\Omega_{2}, f_{2}, p\right)$ and $v_{1}, v_{2}$ are the minimal solutions of (1.5) with $\Omega=\Omega_{1}, \Omega_{2}, f=f_{1}, f_{2}$, respectively, then $v_{1} \leq v_{2}$ in $\Omega_{1}$. If moreover $\Omega=\Omega_{1}=$ $\Omega_{2}$ and $f_{1} \not \equiv f_{2}$, then $v_{1}<v_{2}$ in $\Omega$.

Proof. For any $\lambda<\lambda^{*}\left(\Omega_{2}, f_{2}, p\right)$, let $v_{2}$ be the minimal solution of (1.5) with $\Omega=\Omega_{2}, f=f_{2}$. Then $v_{2}$ is a supersolution of (1.5) with $\Omega=\Omega_{1}, f=f_{1}$, the minimal solution $v_{1}$ of $(1.5)$ satisfies $0 \leq v_{1} \leq v_{2}$ in $\Omega_{1}$. Hence $\lambda^{*}\left(\Omega_{1}, f_{1}, p\right) \geq$ $\lambda^{*}\left(\Omega_{2}, f_{2}, p\right)$.

Suppose that $\Omega=\Omega_{1}=\Omega_{2}$, and $G(x, y)$ be the Green function for $\Delta$ in $\Omega$. Then

$$
v_{i}(x)=\lambda \int_{\Omega} G(x, y) \frac{f_{i}(y)}{\left(L-v_{i}(y)\right)^{p}} d y, \quad \forall i=1,2 .
$$

If $f_{1} \not \equiv f_{2}$, there exists a set $A \subset \Omega$ of positive measure such that $\frac{f_{1}}{\left(L-v_{1}\right)^{p}}<$ $\frac{f_{2}}{\left(L-v_{2}\right)^{p}}$ in A. Then by $(3.2), v_{1}<v_{2}$ in $\Omega$ and the proposition follows.

Theorem 3.4. Assume $f$ satisfies (1.2), $p>1$, then for every $0<\lambda<\lambda^{*}$, there exists a unique global solution $u(x, t)$ of $(1.4)$ which monotonically converges as $t \longrightarrow+\infty$ to the unique minimal solution $u_{*}$ of $(1.5)$. Furthermore, there exists $p_{0}>1$, as a function of $p$, the pull-in voltage $\lambda^{*}(p)$ is smooth, strictly decreasing with respect to $1<p<p_{0}$, and increasing with respect to $p>p_{0}$.

Proof. Fix $0<\lambda<\lambda^{*}$, using the method of $[4,10]$ we can obtain the existence of an unique minimal solution $u_{*}$ of (1.5). It is clear that the pair $u^{-}=0$ and $u^{+}=u_{*}$ are sub- and sup-solutions of (1.4). This implies that the unique global solution $u(x, t)$ of $(1.4)$ satisfies $L>u_{*} \geq u \geq 0$ in $\Omega \times(0, \infty)$.

By differentiating in time and setting $v=u_{t}$, we get for any fixed $T_{0}>0$

$$
\begin{cases}v_{t}=D \Delta v+\frac{p \lambda f(x)}{(L-u)^{p+1}} v, \quad(x, t) \in \Omega \times\left(0, T_{0}\right), \\ v(x, t)=0, & (x, t) \in \partial \Omega \times\left(0, T_{0}\right), \\ v(x, 0) \geq 0, & x \in \Omega .\end{cases}
$$

Here $\frac{p \lambda f(x)}{(L-u)^{p+1}}$ is a locally bounded non-negative function, and by the strong maximum principle, we get that $u_{t}=v>0$ for $(x, t) \in \Omega \times\left(0, T_{0}\right)$. Otherwise $u_{t}=0$, i.e., $u(x, t)=u_{*}$ for any $t>0$. It follows that $u_{t}>0$ holds, and since $u$ is bounded, this monotonicity property implies that the unique global solution 
$u(x, t)$ converges to some function $u^{*}$ as $t \longrightarrow+\infty$. Hence,

$$
0 \leq u^{*} \leq u_{*}<L, \quad x \in \Omega .
$$

The linear stationary boundary problem

$$
\left\{\begin{array}{l}
D \Delta v+\frac{\lambda f(x)}{\left(L-u^{*}\right)^{p}}=0, \quad x \in \Omega \\
v=0, \quad x \in \partial \Omega
\end{array}\right.
$$

Let $\omega(x, t)=u(x, t)-v(x)$. Then $\omega$ satisfies

$$
\left\{\begin{array}{lc}
\omega_{t}-D \Delta \omega=\lambda f(x)\left[\frac{1}{(L-u)^{p}}-\frac{1}{\left(L-u^{*}\right)^{p}}\right],(x, t) \in \Omega \times(0, \infty), \\
\omega(x, t)=0, & (x, t) \in \partial \Omega \times(0, \infty), \\
\omega(x, 0)=-v(x), & x \in \Omega .
\end{array}\right.
$$

By a standard eigenfunction expansion, we imply that the solution $\omega$ of (3.6) also converges to zero in $L^{p}(\Omega)$ as $t \longrightarrow+\infty$. This shows that $u \rightarrow v$ in $L^{p}(\Omega)$ as $t \longrightarrow+\infty$. But since $u \rightarrow u^{*}$ pointwise in $\Omega$ as $t \longrightarrow+\infty$, we deduce that $v(x) \equiv u^{*}(x)$ in $L^{p}(\Omega)$, which implies that $u^{*}(x)$ is also a solution for (1.5). The minimal property of $u_{*}(x)$ then yields that $u_{*}(x) \equiv u^{*}(x)$ on $\Omega$, from which it follows that for every $x \in \Omega$, we have $u(x, t) \downarrow u_{*}$ as $t \longrightarrow+\infty$.

Following Section 2, we define the pull-in voltage $\lambda^{*}$, that is as in Lemma 2.1. We use $\lambda^{*}(p)$ to denote the function

$$
\lambda^{*}(p)=C \frac{(L p)^{p}}{(p+1)^{p+1}},
$$

where $C=\frac{k_{1} D L}{\inf _{x \in \Omega} f(x)}$. However, since

$$
\lambda_{p}^{*}=\lambda^{*}(p) \ln \left(\frac{L p}{p+1}\right) .
$$

Fix $1<L<2$, there exists $p_{0}=\frac{1}{L-1}>1$, we see that there holds

$$
\lambda_{p}^{*}<0, \quad \text { if } 1<p<p_{0},
$$

and

$$
\lambda_{p}^{*}>0, \quad \text { if } \quad p>p_{0} .
$$

Which imply that $\lambda^{*}(p)$ is strictly decreasing with respect to $1<p<p_{0}$, and increasing with respect to $p>p_{0}$. The proof of the theorem is complete.

We know from Lemma 3.2 that there is no solution for (1.5) as soon as $\lambda>\lambda^{*}$. Since the solution $u(x, t)$ of (1.4) is strictly increasing in time $t$, then there must be $T \leq \infty$ such that $u(x, t)$ reaches $L$ at some point of $\bar{\Omega}$ as $t \rightarrow T^{-}$. Otherwise, a proof similar to Theorem 3.4 would imply that $u(x, t)$ will converge to its steady-state which is then the unique minimal solution $u_{*}$ of (1.5), contrary to the hypothesis that $\lambda>\lambda^{*}$. We have:

Corollary 3.5. Assume $f$ satisfies (1.2), $p>1$, if $\lambda>\lambda^{*}$, then the solution $u(x, t)$ of (1.4) must touchdown at a finite time or infinite time. 
Acknowledgments. The authors would like to thank Professor Yisong Yang and the referee for guidance regarding this paper. This work was supported in part by the Natural Science Foundation of Henan Province (112300410054, 12A110004) of China.

\section{References}

[1] P. Esposito, N. Ghoussoub, and Y. Guo, Mathematical analysis of partial differential equations modeling electrostatic MEMS, Courant Lecture Notes in Mathematics 20, New York, 2010.

[2] G. Flores, G. A. Mercado, J. A. Pelesko, and N. Smyth, Analysis of the dynamics and touchdown in a model of electrostatic MEMS, SIAM J. Appl. Math. 67 (2006/2007), no. 2, 434-446.

[3] A. Friedman, Partial Differential Equations of Parabolic Type, Prentice-Hall, Inc., Englewood Cliffs, N.J., U.S.A., 1964.

[4] N. Ghoussoub and Y. Guo, On the partial differential equations of electrostatic MEMS devices: Stationary case, SIAM J. Math. Anal. 38 (2006/07), no. 5, 1423-1449.

[5] _ On the partial differential equations of electrostatic MEMS devices II: Dynamic case, Nonlinear Differential Equations Appl. 15 (2008), no. 1-2, 115-145.

[6] Y. Guo, Z. Pan, and M. Ward, Touchdown and pull-in voltage behavior of a MEMS device with varying dielectric properties, SIAM J. Appl. Math. 66 (2005), no. 1, 309338.

[7] K. M. Hui, Global and touchdown behaviour of the generalized MEMS device equation, Adv. Math. Sci. Appl. 19 (2009), no. 2, 347-370.

[8] — Quenching behaviour of a nonlocal parabolic MEMS equation, http:// arxiv.org/0908.1227v2.

[9] O. A. Ladyzenskaja, V. A. Solonnikov, and N. N. Uralceva, Linear and Quasilinear Equations Of Parabolic Type, Transl. Math. Monographs, Amer. Math. Soc., 23, Providence, R. I. USA, 1968.

[10] F. H. Lin and Y. S. Yang, Nonlinear non-local elliptic equation modelling electrostatic actuation, Proc. R. Soc. A 463 (2007), no. 2081, 1323-1337.

[11] J. A. Pelesko, Mathematical modeling of electrostatic MEMS with tailored dielectric properties, SIAM J. Appl. Math. 62 (2001/2002), no. 3, 888-908.

[12] Z. P. Wang and L. Z. Ruan, On a class of semilinear elliptic problems with singular nonlinearities, Appl. Math. Comput. 193 (2007), no. 1, 89-105.

[13] D. Ye and F. Zhou, On a general family of nonautonomous elliptic and parabolic equations, Calc. Var. Partial Differential Equations 37 (2010), no. 1-2, 259-274.

RUIFENG ZHANG

Institute of CONTEMPorary MATHEMATiCs

HENAN UNIVERSITY

AND

College of Mathematics and Information Science

Henan University

Kaifeng, Henan Province, 475001, P. R. China

E-mail address: zrf615@henu.edu.cn

NA Li

Wan fang College of Science Technology HPU

Zhengzhou, Henan Province, 451400, P. R. China

E-mail address: In2005517@sina.com 